Pacific

Journal of

Mathematics

ON KUMMER TYPE CONSTRUCTION OF SUPERSINGULAR K3 SURFACES IN CHARACTERISTIC 2

ICHIRO SHIMADA AND DE-QI ZHANG 


\title{
ON KUMMER TYPE CONSTRUCTION OF SUPERSINGULAR K3 SURFACES IN CHARACTERISTIC 2
}

\author{
ICHIRO SHIMADA AND DE-QI ZHANG
}

\begin{abstract}
We show that every supersingular $K 3$ surface in characteristic 2 with Artin invariant at most 2 is obtained by the Kummer-type construction of Schröer.
\end{abstract}

\section{Introduction}

We work over an algebraically closed field $k$. A $K 3$ surface $X$ is called supersingular (in the sense of Shioda) if the rank of the Néron-Severi lattice NS $(X)$ of $X$ attains the possible maximum of 22. Supersingular $K 3$ surfaces exist only when char $k$ is positive. The Artin invariant $\sigma(X)$ of a supersingular $K 3$ surface $X$ is defined in [Artin 1974] by

$$
\operatorname{disc} \operatorname{NS}(X)=-p^{2 \sigma(X)},
$$

where $p=$ char $k>0$. It is known that $\sigma(X)$ is a positive integer $\leq 10$.

Let $A$ be an abelian surface, and let $\iota: A \rightarrow A$ be the involution $x \mapsto-x$. If char $k \neq 2$, then the minimal resolution of the quotient surface $A /\langle\iota\rangle$ is a $K 3$ surface, which is called the Kummer surface associated with A.

An abelian surface $A$ in positive characteristic is called supersingular if $A$ is isogenous to a product of supersingular elliptic curves. Ogus [1979; 1983] proved that if char $k>2$, the supersingular $K 3$ surfaces with Artin invariant $\leq 2$ are exactly the Kummer surfaces associated with supersingular abelian surfaces. (See also [Shioda 1979].) On the other hand, Shioda [1974] and Katsura [1978] observed that if char $k=2$, then the minimal resolution of the quotient of a supersingular abelian surface by the involution $x \mapsto-x$ is a rational surface.

Schröer [2007] presented a Kummer type construction of supersingular $K 3$ surfaces in characteristic 2. We assume that char $k=2$ in this paragraph. Let $C \times C$ be the self-product of the rational curve $C$ with one ordinary cusp. We put

$$
\begin{array}{ll}
C=\operatorname{Spec} k\left[u^{2}, u^{3}\right] \cup \operatorname{Spec} k\left[u^{-1}\right] & \text { for the first factor, } \\
C=\operatorname{Spec} k\left[v^{2}, v^{3}\right] \cup \operatorname{Spec} k\left[v^{-1}\right] & \text { for the second factor. }
\end{array}
$$

MSC2000: $14 \mathrm{~J} 28$.

Keywords: supersingular $K 3$ surface, characteristic 2. 
Let $r$ and $s$ be constants in $k$ such that $(r, s) \neq(0,0)$. Then the derivation

$$
\left(u^{-2}+r\right) \frac{\partial}{\partial u}+\left(v^{-2}+s\right) \frac{\partial}{\partial v}
$$

defines a global vector field $\delta$ on $C \times C$ satisfying $\delta^{[2]}=0$. Hence $\delta$ corresponds to an action of the infinitesimal group scheme $\alpha_{2}$ on $C \times C$. Let $X_{r, s}$ be the minimal resolution of the quotient surface $(C \times C) / \alpha_{2}$.

Theorem 1.1 [Schröer 2007]. The surface $X_{r, s}$ is a supersingular K3 surface with Artin invariant

$$
\sigma\left(X_{r, s}\right)= \begin{cases}1, & \text { if } r=0 \text { or } s=0 \text { or } r^{3}=s^{3}, \\ 2, & \text { otherwise. }\end{cases}
$$

The purpose of this paper is to prove:

Theorem 1.2. Let $X^{\prime}$ be a supersingular $K 3$ surface in characteristic 2 with Artin invariant $\leq 2$. Then there exist constants $r, s \in k$ with $(r, s) \neq(0,0)$ such that $X^{\prime}$ is isomorphic to Schröer's Kummer surface $X_{r, s}$.

Even though the moduli curve of marked supersingular $K 3$ surfaces with Artin invariant $\leq 2$ is constructed [Rudakov and Shafarevich 1981; Ogus 1983], it is not separated. Hence the existence of the complete family of Schröer's Kummer surfaces of dimension 1 does not imply Theorem 1.2 immediately.

The main ingredient of the proof is the following structure theorem for NéronSeveri lattices of supersingular $K 3$ surfaces:

Theorem 1.3 [Rudakov and Shafarevich 1981]. Let $X$ and $X^{\prime}$ be supersingular $K 3$ surfaces defined over the same algebraically closed field. If $\sigma(X)=\sigma\left(X^{\prime}\right)$, then the lattices $\mathrm{NS}(X)$ and $\mathrm{NS}\left(X^{\prime}\right)$ are isomorphic.

Indeed, the Néron-Severi lattice $\mathrm{NS}(X)$ of a supersingular $K 3$ surface $X$ in characteristic $p$ is $p$-elementary ([Rudakov and Shafarevich 1981, Theorem in Section 8]; see also [Artin 1974]). If $p=2$, then NS $(X)$ is of type I [Rudakov and Shafarevich 1981, Proposition in Section 5]. Hence the classification theorem of even hyperbolic $p$-elementary lattices [Rudakov and Shafarevich 1981, Theorem in Section 1] implies Theorem 1.3.

We outline the proof of Theorem 1.2. First note that, by [Schröer 2007, Proposition 6.2], if $\sigma\left(X_{r, s}\right)=2$, then Schröer's Kummer surface $X_{r, s}$ is birational to a purely inseparable double cover $Y_{r, s}$ of $\mathbb{P}^{2}$ defined by

$$
w^{2}=x\left(y^{4}+s^{2} y^{2}\right)+y\left(x^{4}+r^{2} x^{2}\right),
$$

which has rational double points of type $4 D_{4}+5 A_{1}$. Let us assume, for simplicity, that the given supersingular $K 3$ surface $X^{\prime}$ is of Artin invariant 2. We choose one of Schröer's Kummer surfaces $X$ with Artin invariant 2 (for example, we put 
$X:=X_{1, s}$ with $\left.s \notin \mathbb{F}_{4}\right)$. Using the isomorphism between $\mathrm{NS}(X)$ and $\mathrm{NS}\left(X^{\prime}\right)$, we can show that $X^{\prime}$ is also birational to a double cover $Y^{\prime}$ of $\mathbb{P}^{2}$ with rational double points of type $4 D_{4}+5 A_{1}$. Using the notion of half-lines and splitting lines, we can show that the covering morphism $Y^{\prime} \rightarrow \mathbb{P}^{2}$ is purely inseparable, and then we can determine the defining equation of $Y^{\prime}$. It turns out that the defining equation of $Y^{\prime}$ is equal to that of $Y_{t, 1}$ for some nonzero constant $t \in k$. Therefore $X^{\prime}$ is isomorphic to Schröer's Kummer surface $X_{t, 1}$.

A surface birational to a purely inseparable cover of $\mathbb{P}^{2}$ is called a Zariski surface, and its basic properties have been studied in [Blass and Lang 1987]. In [Shimada 2004a; 2004b], we showed that every supersingular $K 3$ surface in characteristic 2 is birational to a purely inseparable double cover of $\mathbb{P}^{2}$ with 21 ordinary nodes, and we studied the Néron-Severi lattice of such a surface. Using the results obtained in [Shimada 2004b], we determined in [Shimada 2006] the moduli curve of polarized supersingular $K 3$ surfaces with Artin invariant $\leq 2$ and with 21 ordinary nodes. Schröer [2007] showed that, as $r$ and $s$ varies, his Kummer surfaces $X_{r, s}$ form a smooth family over the projective line Proj $k[\sqrt{r}, \sqrt{s}]$. It would be an interesting problem to investigate the relation between the moduli curve in [Shimada 2006] and Schröer's projective line.

On the other hand, in [Shimada and Zhang 2007], we investigated supersingular $K 3$ surfaces with 10 ordinary cusps. Such supersingular $K 3$ surfaces exist only in characteristic 3. An example is obtained as a purely inseparable triple cover of $\mathbb{P}^{1} \times \mathbb{P}^{1}$. Here, the proof that $Y^{\prime} \rightarrow \mathbb{P}^{2}$ is purely inseparable uses an argument developed in [Shimada and Zhang 2007].

The plan is as follows. In Section 2, we collect some definitions and facts from the lattice theory. The very elementary Lemmas 2.4 and 2.5 play an important role in the proof that $Y^{\prime}$ is purely inseparable over $\mathbb{P}^{2}$. In Section 3, we review some properties of the Néron-Severi lattice of a $K 3$ surface. We then introduce the notion of half-lines and splitting lines for a polarized $K 3$ surface of degree 2 in Section 4. After investigating the purely inseparable double cover $Y_{r, s} \rightarrow \mathbb{P}^{2}$ birational to Schröer's Kummer surface $X_{r, s}$, we prove Theorem 1.2 in Section 6.

\section{Preliminaries on lattices}

A free $\mathbb{Z}$-module $\Lambda$ of finite rank with a nondegenerate symmetric bilinear form

$$
\Lambda \times \Lambda \rightarrow \mathbb{Z}
$$

denoted by $(u, v) \mapsto u v$ is called a lattice. Let $\Lambda$ be a lattice. The dual lattice $\Lambda^{\vee}$ of $\Lambda$ is the $\mathbb{Z}$-module $\operatorname{Hom}(\Lambda, \mathbb{Z})$. Then $\Lambda$ is naturally embedded into $\Lambda^{\vee}$ as a submodule of finite index. The discriminant group of $\Lambda$ is, by definition, the finite 
abelian group $\Lambda^{\vee} / \Lambda$. There exists a unique symmetric bilinear form

$$
\Lambda^{\vee} \times \Lambda^{\vee} \rightarrow \mathbb{Q}
$$

that extends (2-1). An overlattice of $\Lambda$ is a submodule $N$ of $\Lambda^{\vee}$ containing $\Lambda$ such that the bilinear form (2-2) takes values in $\mathbb{Z}$ on $N \times N$. If $\Lambda$ is a sublattice of a lattice $\Lambda^{\prime}$ with finite index, then $\Lambda^{\prime}$ is embedded into $\Lambda^{\vee}$ in a natural way and hence is regarded as an overlattice of $\Lambda$.

We say that $\Lambda$ is even if $u^{2} \in 2 \mathbb{Z}$ holds for every $u \in \Lambda$. The signature $\left(s_{+}, s_{-}\right)$of a lattice $\Lambda$ lists the number of positive and negative eigenvalues of the intersection matrix of $\Lambda$. We say that $\Lambda$ is negative-definite if $s_{+}=0$ and that $\Lambda$ is hyperbolic if $s_{+}=1$. By abuse of language, a positive definite lattice of rank 1 is also called hyperbolic.

Let $\Lambda$ be an even negative-definite lattice. A vector $r \in \Lambda$ is called a root if $r^{2}=-2$. We denote by $\operatorname{Roots}(\Lambda)$ the set of roots in $\Lambda$. We define an equivalence relation $\sim$ on $\operatorname{Roots}(\Lambda): r \sim r^{\prime}$ if there exists a sequence $r_{0}=r, r_{1}, \ldots, r_{m-1}, r_{m}=$ $r^{\prime}$ of roots in $\Lambda$ such that $r_{i} r_{i+1} \neq 0$ for $i=0, \ldots, m-1$. Let $R_{1}, \ldots, R_{k}$ be the equivalence classes of $\sim$. We call the decomposition

$$
\operatorname{Roots}(\Lambda)=R_{1} \sqcup \cdots \sqcup R_{k}
$$

the irreducible decomposition of $\operatorname{Roots}(\Lambda)$. Suppose that we are given a linear form

$$
\alpha: \Lambda \rightarrow \mathbb{R}
$$

such that $\alpha(r) \neq 0$ for any $r \in \operatorname{Roots}(\Lambda)$. We put

$$
R_{i}^{+}:=\left\{r \in R_{i} \mid \alpha(r)>0\right\} .
$$

A root $r \in R_{i}^{+}$is called decomposable if there exist $r_{1}, r_{2} \in R_{i}^{+}$such that $r=r_{1}+r_{2}$, and $r$ is called indecomposable if it is not decomposable. For the proof of the following results, see [Bourbaki 1981] or [Ebeling 2002], for example.

Proposition 2.1. Let $r$ be an element of $R_{i}^{+}$. Then $r$ can be written uniquely as a linear combination of indecomposable elements of $R_{i}^{+}$. Moreover the coefficients are all nonnegative integers.

Proposition 2.2. Let $\Lambda_{i}$ be the sublattice of $\Lambda$ generated by the roots in $R_{i}$. Then $\Lambda_{1}, \ldots, \Lambda_{k}$ form an orthogonal direct sum in $\Lambda$. The indecomposable elements of $R_{i}^{+}$form a basis of the lattice $\Lambda_{i}$, and the intersection matrix of $\Lambda_{i}$ in this basis is a Cartan matrix of type ADE multiplied by -1 .

The indecomposable elements of $R_{i}^{+}$have the following characterization:

Corollary 2.3. Let $\varepsilon_{1}, \ldots, \varepsilon_{d}$ be elements of $R_{i}^{+}$such that every element of $R_{i}^{+}$ is written uniquely as a linear combination of $\varepsilon_{1}, \ldots, \varepsilon_{d}$ with nonnegative integer 
coefficients. Then $\left\{\varepsilon_{1}, \ldots, \varepsilon_{d}\right\}$ is equals to the set of indecomposable elements of $R_{i}^{+}$.

Proof. Suppose that $\varepsilon_{i}$ is decomposable. There exist $r_{1}, r_{2} \in R_{i}^{+}$such that $\varepsilon_{i}=$ $r_{1}+r_{2}$. Since each of $r_{1}$ and $r_{2}$ is written as a linear combination of $\varepsilon_{1}, \ldots, \varepsilon_{d}$ with nonnegative integer coefficients, we obtain a contradiction to the uniqueness of writing $\varepsilon_{i}$ as a linear combination of $\varepsilon_{1}, \ldots, \varepsilon_{d}$ with nonnegative integer coefficients. Therefore each of $\varepsilon_{1}, \ldots, \varepsilon_{d}$ is indecomposable.

Suppose that $r \in R_{i}^{+}$is indecomposable. We can write $r$ as a linear combination of $\varepsilon_{1}, \ldots, \varepsilon_{d}$ with nonnegative integer coefficients. Since each $\varepsilon_{i}$ is indecomposable, the uniqueness of writing $r$ as a linear combination of indecomposable elements of $R_{i}^{+}$with nonnegative integer coefficients implies that $r$ is equal to one of $\varepsilon_{1}, \ldots, \varepsilon_{d}$.

Let $\tau_{i}$ be the $A D E$-type of the Cartan matrix of the intersection matrix of $\Lambda_{i}$ given in Proposition 2.2. We define the root type of $\Lambda$ to be the formal sum $\tau_{1}+\cdots+\tau_{k}$.

We say that $\Lambda$ is a root lattice if $\Lambda$ is generated by $\operatorname{Roots}(\Lambda)$. For later use, we present properties of root lattices of type $A_{1}$ and $D_{4}$.

Let $\Lambda$ be the root lattice of type $A_{1}$, and let $a \in \Lambda$ be a root that generates $\Lambda$. We put $a^{\vee}:=-a / 2$, which generates $\Lambda^{\vee}$. Then the discriminant group of $\Lambda$ is isomorphic to $\mathbb{Z} / 2 \mathbb{Z}$. The proof of the following is elementary:

Lemma 2.4. Let $v \in \Lambda^{\vee}$ be a vector such that $v a \geq 0$. If $v \equiv 0 \bmod \Lambda$, then we have $v^{2}=0$ or $v^{2} \leq-2$, and $v^{2}=0$ holds if and only if $v=0$. If $v \equiv a^{\vee} \bmod \Lambda$, then we have $v^{2}=-1 / 2$ or $v^{2} \leq-9 / 2$, and $v^{2}=-1 / 2$ holds if and only if $v=a^{\vee}$.

Let $\Lambda$ be the root lattice of type $D_{4}$ generated by the roots $d_{1}, \ldots, d_{4}$ whose intersection numbers are given by the Dynkin diagram in Figure 1 . Let $d_{1}^{\vee}, \ldots, d_{4}^{\vee}$ be the basis of $\Lambda^{\vee}$ dual to $d_{1}, \ldots, d_{4}$. We have

$$
\left[d_{1}^{\vee}, d_{2}^{\vee}, d_{3}^{\vee}, d_{4}^{\vee}\right]=\left[d_{1}, d_{2}, d_{3}, d_{4}\right]\left[\begin{array}{cccc}
-1 & -1 / 2 & -1 & -1 / 2 \\
-1 / 2 & -1 & -1 & -1 / 2 \\
-1 & -1 & -2 & -1 \\
-1 / 2 & -1 / 2 & -1 & -1
\end{array}\right] .
$$

The discriminant group of $\Lambda$ is isomorphic to $(\mathbb{Z} / 2 \mathbb{Z}) \oplus(\mathbb{Z} / 2 \mathbb{Z})$ and is generated by $d_{1}^{\vee} \bmod \Lambda$ and $d_{4}^{\vee} \bmod \Lambda$.

Lemma 2.5. Let $v \in \Lambda^{\vee}$ be a vector such that $v d_{i} \geq 0$ holds for $i=1, \ldots, 4$. If $v \equiv 0 \bmod \Lambda$, then we have $v^{2}=0$ or $v^{2} \leq-2$, and $v^{2}=0$ holds if and only if $v=0$. If $v \equiv d_{1}^{\vee} \bmod \Lambda$, then we have $v^{2}=-1$ or $v^{2} \leq-3$, and $v^{2}=-1$ holds if and only if $v=d_{1}^{\vee}$. 


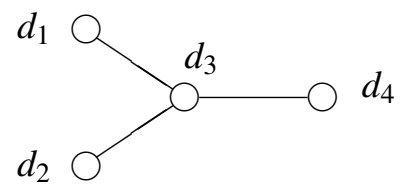

Figure 1. The Dynkin diagram of type $D_{4}$.

Proof. The first assertion is obvious. Suppose that $v \equiv d_{1}^{\vee} \bmod \Lambda$. Then we can put

$$
v=d_{1}^{\vee}+x_{1} d_{1}+x_{2} d_{2}+x_{3} d_{3}+x_{4} d_{4},
$$

where $x_{1}, \ldots, x_{4} \in \mathbb{Z}$. From the condition $v d_{i} \geq 0$ for $i=1, \ldots, 4$, we obtain inequalities:

$$
\begin{aligned}
1-2 x_{1}+x_{3} & \geq 0, \quad-2 x_{2}+x_{3} \geq 0, \\
x_{1}+x_{2}-2 x_{3}+x_{4} & \geq 0, \quad x_{3}-2 x_{4} \geq 0 .
\end{aligned}
$$

Using (2-4), we calculate

$$
\begin{aligned}
v^{2} & =-1-2\left(x_{1}^{2}+x_{2}^{2}+x_{3}^{2}+x_{4}^{2}-x_{1}-x_{1} x_{3}-x_{2} x_{3}-x_{3} x_{4}\right) \\
& =-1-\left\{\left(1-2 x_{1}+x_{3}\right)^{2}+\left(-2 x_{2}+x_{3}\right)^{2}+\left(x_{3}-2 x_{4}\right)^{2}+\left(x_{3}-1\right)^{2}-2\right\} / 2 .
\end{aligned}
$$

Therefore $v^{2}$ is a negative odd integer, and $v^{2}=-1$ holds if and only if two of the four integers $1-2 x_{1}+x_{3},-2 x_{2}+x_{3}, x_{3}-2 x_{4}, x_{3}-1$ are \pm 1 and the other two are 0 . Combining this with the inequalities (2-5), we see that $v^{2}=-1$ holds if and only if $x_{1}=x_{2}=x_{3}=x_{4}=0$.

\section{The Néron-Severi lattice of a $K 3$ surface}

In this section, we work over an algebraically closed field of arbitrary characteristic. Let $X$ be an (algebraic) $K 3$ surface, and let $\mathrm{NS}(X)$ be the Néron-Severi lattice of $X$, which is an even hyperbolic lattice. For a divisor $D$ on $X$, we denote by $[D] \in \mathrm{NS}(X)$ the class of $D$.

3A. The nef-cone. We put

$\operatorname{Nef}(X):=\{v \in \operatorname{NS}(X) \otimes \mathbb{R} \mid v[D] \geq 0$ for any effective divisor $D$ on $X\}$.

Let $A$ be an ample divisor on $X$, and let $\mathscr{C}^{+}(X)$ be the connected component of

$$
\left\{v \in \mathrm{NS}(X) \otimes \mathbb{R} \mid v^{2}>0\right\}
$$

that contains $[A]$. For a vector $v \in \mathrm{NS}(X)$, we put

$$
\langle v\rangle_{\mathbb{R}}^{\perp}:=\{w \in \mathrm{NS}(X) \otimes \mathbb{R} \mid v w=0\} .
$$


Then the family of hyperplanes $\left\{\langle r\rangle_{\mathbb{R}}^{\perp} \mid r^{2}=-2\right\}$ of $\mathrm{NS}(X) \otimes \mathbb{R}$ is locally finite in $\mathscr{C}^{+}(X)$. It is well known and easy to prove that $[A] \notin\langle r\rangle \frac{\perp}{\mathbb{R}}$ for any vector $r$ with $r^{2}=-2$ and that $\operatorname{Nef}(X)$ is equal to the closure in $\operatorname{NS}(X) \otimes \mathbb{R}$ of the connected component of

$$
\mathscr{C}^{+}(X) \backslash \bigcup\langle r\rangle_{\mathbb{R}}^{\perp}
$$

that contains $[A]$. By the argument of Proposition 3 in [Rudakov and Shafarevich 1981, Section 3], we obtain:

Proposition 3.1. Let $X$ and $X^{\prime}$ be two algebraic $K 3$ surfaces such that $\mathrm{NS}(X)$ and $\mathrm{NS}\left(X^{\prime}\right)$ are isomorphic. Then there exists an isomorphism $\phi: \mathrm{NS}(X) \stackrel{\sim}{\rightarrow} \mathrm{NS}\left(X^{\prime}\right)$ such that $\phi \otimes \mathbb{R}$ maps $\operatorname{Nef}(X)$ to $\operatorname{Nef}\left(X^{\prime}\right)$.

\section{B. Polarizations.}

Proposition 3.2. Let $H$ be a divisor on an algebraic $K 3$ surface $X$ such that $[H] \in \operatorname{Nef}(X)$ and $H^{2}>0$. The following conditions are equivalent:

(i) The complete linear system $|H|$ has no fixed components.

(ii) There are no vectors $e \in \mathrm{NS}(X)$ such that $e[H]=1$ and $e^{2}=0$.

Proof. That (i) $\Rightarrow$ (ii) follows from the proof that $(4) \Rightarrow$ (1) in [Urabe 1988, Proposition 1.7], and (ii) $\Rightarrow$ (i) follows from [Nikulin 1991, Proposition 0.1].

Definition 3.3. A polarization of an algebraic $K 3$ surface $X$ is a divisor $H$ on $X$ satisfying $[H] \in \operatorname{Nef}(X), H^{2}>0$ and the conditions (i) and (ii) in Proposition 3.2. The positive integer $H^{2}$ is called the degree of the polarization $H$. By [Nikulin 1991, Proposition 0.1], if $H$ is a polarization of degree $d$, then $|H|$ is base-point free by Saint-Donat [1974, Corollary 3.2], and we have $\operatorname{dim}|H|=1+d / 2$.

A pair $(X, H)$ of a $K 3$ surface $X$ and a polarization $H$ of $X$ is called a polarized K3 surface.

Combining Propositions 3.1 and 3.2, we obtain the following:

Corollary 3.4. Let $X$ and $X^{\prime}$ be two $K 3$ surfaces such that $\operatorname{NS}(X)$ and $\operatorname{NS}\left(X^{\prime}\right)$ are isomorphic, and let $H$ be a polarization of $X$. If $\phi: \operatorname{NS}(X) \stackrel{\sim}{\rightarrow} \mathrm{NS}\left(X^{\prime}\right)$ is an isomorphism such that $\phi \otimes \mathbb{R}$ maps $\operatorname{Nef}(X)$ to $\operatorname{Nef}\left(X^{\prime}\right)$, then $\phi([H])$ is the class of a polarization $H^{\prime}$ of $X^{\prime}$.

A curve $C$ on $X$ is called a (-2)-curve on $X$ if it satisfies the equivalent conditions:

(i) $C$ is a smooth rational curve;

(ii) $C$ is reduced irreducible with negative self-intersection;

(iii) $C$ is irreducible and $C^{2}=-2$. 
Let $(X, H)$ be a polarized $K 3$ surface. Then the complete linear system $|H|$ defines a morphism $\Phi_{|H|}$ from $X$ to a projective space $\mathbb{P}^{N}\left(N=1+H^{2} / 2\right)$ that is generically finite over the image. We denote by

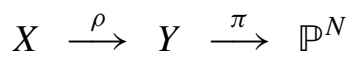

the Stein factorization of $\Phi_{|H|}$; that is, $\rho$ is birational, $Y$ is normal, and $\pi$ is finite. The normal $K 3$ surface $Y$ has only rational double points as its singularities, and hence $\rho$ is a contraction of an $A D E$-configuration of (-2)-curves [Artin 1962; 1966]. Let $\mathscr{E}$ be the set of $(-2)$-curves that are contracted by $\rho$. The classes $[E]$ of $E \in \mathscr{E}$ are determined by the following procedure. Let $[H]^{\perp}$ be the orthogonal complement of $[H]$ in $\operatorname{NS}(X)$. Since $\operatorname{NS}(X)$ is even hyperbolic and $[H]^{2}$ is positive, $[H]^{\perp}$ is even and negative-definite. We can therefore consider the set $\operatorname{Roots}\left([H]^{\perp}\right)$ of roots in $[H]^{\perp}$.

Lemma 3.5. Let $r$ be an element of $\operatorname{Roots}\left([H]^{\perp}\right)$. Then there exists a unique effective divisor $E$ such that $r=[E]$ or $r=-[E]$ holds. Moreover, the integral component of $E$ is a (-2)-curve.

Proof. By the Riemann-Roch theorem and the Serre duality, we see that either $r$ or $-r$ is the class of an effective divisor. Replacing $r$ with $-r$, if necessary, we can assume that $r$ is the class of an effective divisor $E$. Let $E=F+M$ be the decomposition of $E$ into the sum of the fixed part $F$ and the movable part $M$. Since $[H] \in \operatorname{Nef}(X)$, we have $H F \geq 0$ and $H M \geq 0$. Because $H E=0$, we have $H M=0$. Since $[H]^{\perp}$ is negative-definite and $M^{2} \geq 0$, we get $M=0$. Therefore $E$ is unique and every irreducible component of $E$ has negative self-intersection number. Thus the reduced part of every irreducible component of $E$ is a (-2)-curve.

Let Roots $\left([H]^{\perp}\right)=R_{1} \sqcup \cdots \sqcup R_{k}$ be the irreducible decomposition of Roots $\left([H]^{\perp}\right)$ defined in Section 2. We choose an interior point $a$ of $\operatorname{Nef}(X)$ (for example, the class of an ample divisor on $X)$, and let $\alpha: \operatorname{NS}(X) \rightarrow \mathbb{R}$ be the linear form given by $\alpha(x):=a x$. By Lemma 3.5, we see that $\alpha(r) \neq 0$ for any $r \in \operatorname{Roots}\left([H]^{\perp}\right)$. We thus can define $R_{i}^{+}$by (2-3) and consider the indecomposable roots of $R_{i}^{+}$. Note that $R_{i}^{+} \subset R_{i}$ does not depend on the choice of the interior point $a$ of $\operatorname{Nef}(X)$.

Proposition 3.6. Let $\operatorname{Sing}(Y)$ be the set of singular points of $Y$. There exists a bijection from the set $\left\{R_{1}, \ldots, R_{k}\right\}$ to $\operatorname{Sing}(Y)$ with the following property. Let $P_{i} \in \operatorname{Sing}(Y)$ be the point corresponding to $R_{i}$. Then the classes of $(-2)$-curves contracted by $\rho$ to $P_{i}$ are exactly the indecomposable roots of $R_{i}^{+}$.

Proof. Let $r$ be an element of $R_{i}^{+}$. By Lemma 3.5 and $\alpha(r)>0, r$ is the class of a unique effective divisor of the form $a_{1} E_{1}+\cdots+a_{l} E_{l}$, where $E_{1}, \ldots, E_{l}$ are $(-2)$-curves and $a_{1}, \ldots, a_{l}$ are positive integers. Since $[H] \in \operatorname{Nef}(X)$ and $r \in[H]^{\perp}$, we have $\left[E_{v}\right] \in[H]^{\perp}$ for $v=1, \ldots, l$. In particular, we have $E_{v} \in \mathscr{E}$ 
for $v=1, \ldots, l$. Let $\Lambda_{j}$ be the sublattice of $[H]^{\perp}$ generated by the roots in $R_{j}$ for $j=1, \ldots, k$. Since $\Lambda_{1}, \ldots, \Lambda_{k}$ form a direct sum in $\operatorname{NS}(X)$, the uniqueness of the effective divisor representing $r \in \Lambda_{i}$ implies that $\left[E_{1}\right], \ldots,\left[E_{l}\right]$ are all in $R_{i}$. Since $\alpha\left(\left[E_{v}\right]\right)>0$, we have $\left[E_{v}\right] \in R_{i}^{+}$. Thus we have shown that every element of $R_{i}^{+}$is uniquely written as a linear combination of the classes of $(-2)$-curves in $R_{i}^{+}$with nonnegative integer coefficients. By Corollary 2.3, we see that $r$ is the class of a (-2)-curve in $\mathscr{E}$ if and only if $r$ is indecomposable in $R_{i}^{+}$.

Let $\left(X^{\prime}, H^{\prime}\right)$ be another polarized $K 3$ surface. Let

$$
X^{\prime} \stackrel{\rho^{\prime}}{\longrightarrow} Y^{\prime} \stackrel{\pi^{\prime}}{\longrightarrow} \mathbb{P}^{N^{\prime}}
$$

be the Stein factorization of the morphism $\Phi_{\left|H^{\prime}\right|}$ defined by $\left|H^{\prime}\right|$, and let $\mathscr{E}^{\prime}$ be the set of $(-2)$-curves contracted by $\rho^{\prime}$.

Corollary 3.7. Suppose that there exists an isomorphism $\phi: \operatorname{NS}(X) \stackrel{\sim}{\rightarrow} \operatorname{NS}\left(X^{\prime}\right)$ such that $\phi \otimes \mathbb{R}$ maps $\operatorname{Nef}(X)$ to $\operatorname{Nef}\left(X^{\prime}\right)$ and that $\phi([H])$ is equal to $\left[H^{\prime}\right]$. Then the ADE-type of $\operatorname{Sing}(Y)$ coincides with that of $\operatorname{Sing}\left(Y^{\prime}\right)$. Moreover, there exist bijections

$$
\phi_{\mathscr{E}}: \stackrel{\mathscr{C}}{\mathscr{\sim}} \rightarrow \mathscr{C}^{\prime} \quad \text { and } \quad \phi_{\text {Sing }}: \operatorname{Sing}(Y) \stackrel{\sim}{\rightarrow} \operatorname{Sing}\left(Y^{\prime}\right)
$$

such that the following diagram is commutative:

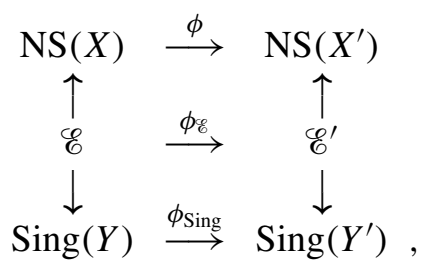

where the up-arrows are given by $E \mapsto[E] \in \mathrm{NS}(X)$ and $E^{\prime} \mapsto\left[E^{\prime}\right] \in \mathrm{NS}\left(X^{\prime}\right)$, respectively, and the down-arrows are given by $E \mapsto \rho(E) \in \operatorname{Sing}(Y)$ and $E^{\prime} \mapsto$ $\rho^{\prime}\left(E^{\prime}\right) \in \operatorname{Sing}\left(Y^{\prime}\right)$, respectively.

\section{C. Polarizations with maximal rational double points.}

Definition 3.8. We say that a polarized $K 3$ surface $(X, H)$ has maximal rational double points if the total Milnor number of $\operatorname{Sing}(Y)$ is equal to $\operatorname{rank} \operatorname{NS}(X)-1$ or, equivalently, if $\operatorname{Roots}\left([H]^{\perp}\right)$ generates a root lattice of finite index in $[H]^{\perp}$.

Let $(X, H)$ be a polarized $K 3$ surface with maximal rational double points. Consider the Stein factorization (3-1) of $\Phi_{|H|}$. For $P \in \operatorname{Sing}(Y)$, we denote by $\mathscr{E}_{P} \subset \mathscr{E}$ the set of (-2)-curves that are contracted to $P$ by $\rho$, by $\Lambda_{P} \subset \mathrm{NS}(X)$ the sublattice generated by the classes $[E]$ of the curves $E \in \mathscr{E}_{P}$, and by $\Delta_{P}$ the discriminant group $\Lambda_{P}^{\vee} / \Lambda_{P}$ of $\Lambda_{P}$. We also denote by $\Lambda_{H} \subset \mathrm{NS}(X)$ the sublattice of rank 1 
generated by $[H]$ and by $\Delta_{H}$ the discriminant group $\Lambda_{H}^{\vee} / \Lambda_{H}$ of $\Lambda_{H}$, which is a cyclic group of order equal to $H^{2}$. We then put

$$
\Lambda:=\Lambda_{H} \oplus \bigoplus_{P \in \operatorname{Sing}(Y)} \Lambda_{P} \quad \text { and } \quad \Delta:=\Lambda^{\vee} / \Lambda
$$

We have natural decompositions

$$
\Lambda^{\vee}=\Lambda_{H}^{\vee} \oplus \bigoplus_{P \in \operatorname{Sing}(Y)} \Lambda_{P}^{\vee} \quad \text { and } \quad \Delta=\Delta_{H} \oplus \bigoplus_{P \in \operatorname{Sing}(Y)} \Delta_{P} .
$$

By the assumption, $\Lambda$ is of finite index in $\mathrm{NS}(X)$, and hence $\mathrm{NS}(X)$ is an overlattice of $\Lambda$. Let $v$ be a vector of $\operatorname{NS}(X)$. Using the direct-sum decomposition of $\Lambda^{\vee}$ and the natural embedding $\mathrm{NS}(X) \hookrightarrow \Lambda^{\vee}$, we can define the $H$-component $v_{H} \in \Lambda_{H}^{\vee}$ and the $P$-components $v_{P} \in \Lambda_{P}^{\vee}$ of $v$. We denote by $\bar{v} \in \Delta$ the class of $v$ modulo $\Lambda$. Then the $H$-component $\bar{v}_{H} \in \Delta_{H}$ and the $P$-components $\bar{v}_{P} \in \Delta_{P}$ of $\bar{v}$ are also defined.

\section{Polarizations of degree 2 in characteristic 2}

From now on, we assume that the base field $k$ is of characteristic 2 .

Let $(X, H)$ be a polarized $K 3$ surface of degree 2. Then the Stein factorization of $\Phi_{|H|}$ is of the form

$$
X \stackrel{\rho}{\longrightarrow} Y \stackrel{\pi}{\longrightarrow} \mathbb{P}^{2},
$$

where $\pi: Y \rightarrow \mathbb{P}^{2}$ is a finite double cover. We have $h^{0}\left(X, \mathcal{O}_{X}(m H)\right)=m^{2}+2$ for every $m \geq 1$ by [Nikulin 1991, Proposition 0.1]. Therefore the finite double cover $\pi: Y \rightarrow \mathbb{P}^{2}$ is defined by the equation

$$
w^{2}+w C\left(x_{0}, x_{1}, x_{2}\right)+G\left(x_{0}, x_{1}, x_{2}\right)=0
$$

in the total space of the line bundle $V \rightarrow \mathbb{P}^{2}$ corresponding to the invertible sheaf $\mathrm{O}_{\mathbb{P}^{2}}(3)$, where $w$ is a fiber coordinate of $V,\left[x_{0}: x_{1}: x_{2}\right]$ is a homogeneous coordinate system of $\mathbb{P}^{2}$, and $C$ and $G$ are homogeneous polynomials of degree 3 and 6 that are regarded as sections of $V$ and $V^{\otimes 2}$, respectively. If $C \neq 0$, then $\pi$ is separable, while if $C=0$, then $\pi$ is purely inseparable.

Definition 4.1. An irreducible curve $F \subset X$ is called a half-line of $(X, H)$ if $F H=1$ holds. A line $L \subset \mathbb{P}^{2}$ is said to be splitting in $(X, H)$ if the proper transform of $L$ in $X$ is nonreduced or reducible, or equivalently, if the schemetheoretic preimage $\pi^{-1}(L) \subset Y$ of $L$ by $\pi$ is nonreduced or reducible.

Let $F$ be a half-line of $(X, H)$. Then $\Phi_{|H|}$ induces an isomorphism from $F$ to a line $L \subset \mathbb{P}^{2}$, and this line $L$ is splitting in $(X, H)$. In particular, a half-line is a $(-2)$-curve. 
Definition 4.2. If $L \subset \mathbb{P}^{2}$ is a line splitting in $(X, H)$, then the proper transform of $L$ in $X$ is written as $F+F^{\prime}$, where $F$ and $F^{\prime}$ are half-lines of $(X, H)$. These halflines are said to be lying over $L$. We say that $L$ is of nonreduced type if $F=F^{\prime}$, while $L$ is of reduced type if $F \neq F^{\prime}$.

Lemma 4.3. Suppose that $\pi$ is separable. Then the number of splitting lines of nonreduced type is at most 3.

Proof. Let $L$ be a splitting line of nonreduced type. We choose homogeneous coordinates $\left[x_{0}: x_{1}: x_{2}\right]$ of $\mathbb{P}^{2}$ such that $L$ is defined by $x_{2}=0$. Putting $x_{2}=0$ in the defining Equation (4-1), we see that the curve defined by

$$
w^{2}+w C\left(x_{0}, x_{1}, 0\right)+G\left(x_{0}, x_{1}, 0\right)=0
$$

in the total space of the line bundle $\left.V\right|_{L} \rightarrow L$ on $L$ is nonreduced. Let $\gamma\left(w, x_{0}, x_{1}\right)$ be the left-hand side of (4-2). Since char $k=2$, we have $\partial \gamma / \partial w=C\left(x_{0}, x_{1}, 0\right)$. Therefore $C\left(x_{0}, x_{1}, 0\right)$ is constantly equal to zero. Thus we have shown that the defining equation of a splitting line of nonreduced type divides $C\left(x_{0}, x_{1}, x_{2}\right)$. Thus, if $C \neq 0$, then the number of splitting lines of nonreduced type is at most $\operatorname{deg} C=3$.

Next we investigate the case where $\pi$ is purely inseparable. Then $\pi$ is given by the equation

$$
w^{2}+G\left(x_{0}, x_{1}, x_{2}\right)=0 .
$$

Note that every splitting line is now of nonreduced type.

Remark 4.4. Let $\Gamma\left(x_{0}, x_{1}, x_{2}\right)$ be a homogeneous polynomial of degree 3 . Then the equations $w^{2}=G$ and $w^{2}=G+\Gamma^{2}$ define surfaces isomorphic over $\mathbb{P}^{2}$.

We have the following relation between splitting lines and rational double points of $Y$. See [Artin 1977] or [Greuel and Kröning 1990] for the normal form of defining equations of rational double points in characteristic 2 .

Lemma 4.5. Let $L \subset \mathbb{P}^{2}$ be a line defined by $\ell\left(x_{0}, x_{1}, x_{2}\right)=0$.

(1) The line $L$ is splitting in $(X, H)$ if and only if there exist homogeneous polynomials $Q\left(x_{0}, x_{1}, x_{2}\right)$ and $\Gamma\left(x_{0}, x_{1}, x_{2}\right)$ of degree 5 and 3 , respectively, such that $G=\ell Q+\Gamma^{2}$.

(2) Suppose that $L$ is splitting in $(X, H)$, and let $Q$ be a polynomial of degree 5 such that $G+\ell Q$ is a square of a cubic polynomial. We denote by $T \subset \mathbb{P}^{2}$ the quintic curve defined by $Q=0$. Let $p$ be a point of $L$, and $P$ the point of $Y$ such that $\pi(P)=p$. Then $P$ is a smooth point of $Y$ if and only if $p \notin T$, and $P$ is an $A_{1}$-singular point of $Y$ if and only if $T$ intersects $L$ transversely at $p$. 
Proof. We can assume that $\ell=x_{2}$. Since the curve defined by $w^{2}+G\left(x_{0}, x_{1}, 0\right)=0$ in $\left.V\right|_{L}$ is nonreduced, we see that $G\left(x_{0}, x_{1}, 0\right)$ is the square of a polynomial of degree 3. Hence the claim (1) follows. Let $(x, y)$ be an affine coordinate system of $\mathbb{P}^{2}$ with the origin $p$ such that $L$ is defined by $y=0$. We write (4-3) as $w^{2}=g(x, y)$. Let $g_{i j}$ be the coefficient of $x^{i} y^{j}$ in $g$. Then $P$ is a smooth point of $Y$ if and only if $g_{01} \neq 0$ or $g_{10} \neq 0$, and $P$ is an $A_{1}$-singular point of $Y$ if and only if $g_{01}=g_{10}=0$ and $g_{11} \neq 0$. Let $q(x, y)$ be the inhomogeneous polynomial corresponding to $Q$, and let $q_{i j}$ be the coefficients of $x^{i} y^{j}$ in $q$. Then, up to a multiplicative constant, we have $g_{01}=q_{00}, g_{10}=0, g_{11}=q_{10}$. Therefore the claim (2) follows.

Remark 4.6. The polynomials $Q$ and $\Gamma$ such that $G=\ell Q+\Gamma^{2}$ are not determined uniquely by $G$ and $\ell$. However, the homogeneous polynomial $Q \mid L$ on the line $L$ is determined uniquely by $G$ and $\ell$.

\section{Schröer's Kummer surfaces as Zariski surfaces}

Let $r$ and $s$ be constants in $k$ such that $r \neq 0, s \neq 0$ and $r^{3} \neq s^{3}$. Then Schröer's supersingular $K 3$ surface $X_{r, s}$, defined in the introduction, is of Artin invariant 2. By Proposition 6.2 of [Schröer 2007], the quotient surface $(C \times C) / \alpha_{2}$ of the $\alpha_{2}$-action on $C \times C$ defined by the vector field (1-1) contains an open subset $U$ isomorphic to

$$
\operatorname{Spec} k[a, b, c] /\left(c^{2}+a\left(b^{4}+s^{2} b^{2}\right)+b\left(a^{4}+r^{2} a^{2}\right)\right) .
$$

The singular locus of $U$ consists of four $D_{4}$-singular points coming from the fixed points of the $\alpha_{2}$-action on the smooth part of $C \times C$. Let

$$
\pi_{r, s}: Y_{r, s} \rightarrow \mathbb{P}^{2}
$$

be the purely inseparable double cover defined by

$$
w^{2}=\left[x_{0}\left(x_{1}^{4}+s^{2} x_{1}^{2} x_{2}^{2}\right)+x_{1}\left(x_{0}^{4}+r^{2} x_{0}^{2} x_{2}^{2}\right)\right] x_{2},
$$

which is a projective completion of $U$. Then $Y_{r, s}$ is birational to $X_{r, s}$, and hence there exists a morphism $\rho_{r, s}: X_{r, s} \rightarrow Y_{r, s}$ that is the minimal resolution. The pull-back of a line of $\mathbb{P}^{2}$ by $\pi_{r, s} \circ \rho_{r, s}$ is a polarization $H_{r, s}$ of degree 2 of $X_{r, s}$. Then

$$
X_{r, s} \stackrel{\rho_{r, s}}{\longrightarrow} Y_{r, s} \stackrel{\pi_{r, s}}{\longrightarrow} \mathbb{P}^{2}
$$

is the Stein factorization of $\Phi_{\left|H_{r, s}\right|}$. The singular locus of $Y_{r, s}$ consists of four $D_{4}$-singular points $P(00), P(01), P(10), P(11)$ in $U$ and five $A_{1}$-singular points $Q(0), Q(1), Q(\omega), Q(\bar{\omega}), Q(\infty)$ lying on the line defined by $x_{2}=0$. Here $\omega$ is a primitive third root of 1 , and $\bar{\omega}=\omega^{2}$. These singular points are indexed so that their images by $\pi_{r, s}$ are given in Table 1 , where $p(\alpha \beta):=\pi_{r, s}(P(\alpha \beta))$ for $\alpha \beta=00,01,10,11$, and $q(\gamma):=\pi_{r, s}(Q(\gamma))$ for $\gamma=0,1, \omega, \bar{\omega}, \infty$. It is easy to 


$$
\begin{array}{l|rl}
p(00)=[0: 0: 1] & q(0)=[1: 0: 0] \\
p(01)=[0: s: 1] & q(1)=[1: 1: 0] \\
p(10)=[r: 0: 1] & q(\omega)=[1: \omega: 0] \\
p(11)=[r: s: 1] & q(\bar{\omega})=[1: \bar{\omega}: 0] \\
& q(\infty)=[0: 1: 0]
\end{array}
$$

Table 1. The coordinates of the singular points of $Y_{r, s}$.

see that the five lines listed below are splitting in $\left(X_{r, s}, H_{r, s}\right)$ :

$$
\begin{array}{ll}
L(\infty):=\left\{x_{2}=0\right\}, & \\
L(0 *):=\left\{x_{0}=0\right\}, & L(1 *):=\left\{x_{0}+r x_{2}=0\right\}, \\
L(* 0):=\left\{x_{1}=0\right\}, & L(* 1):=\left\{x_{1}+s x_{2}=0\right\} .
\end{array}
$$

To simplify the notation, we put

$$
\mathscr{P}:=\{00,01,10,11\}, \quad \mathscr{2}:=\{0,1, \omega, \bar{\omega}, \infty\}, \quad \mathscr{L}:=\{\infty, 0 *, 1 *, * 0, * 1\} .
$$

Figure 2 gives the configuration of the splitting lines $L(\lambda)(\lambda \in \mathscr{L})$ and the points $p(\alpha \beta)(\alpha \beta \in \mathscr{P})$ and $q(\gamma)(\gamma \in \mathscr{2})$. For a splitting line $L(\lambda)(\lambda \in \mathscr{L})$, we denote by

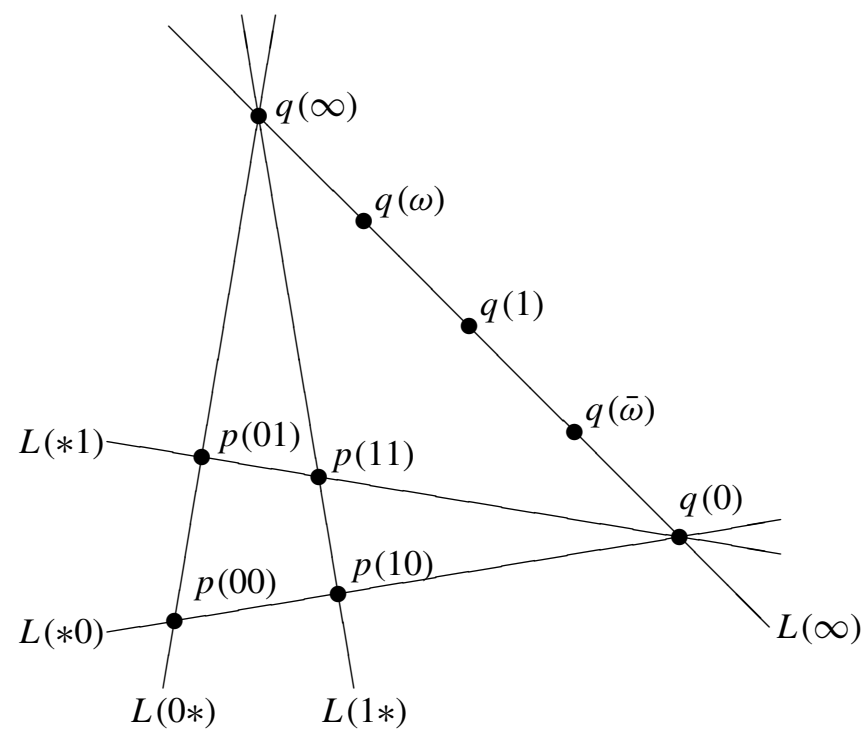

Figure 2. The configuration of splitting lines. 
$F(\lambda)$ the half-line of $\left(X_{r, s}, H_{r, s}\right)$ lying over $L(\lambda)$. By explicitly blowing up $Y_{r, s}$ at their singular points, we see that the half-lines $F(\lambda)$ and the exceptional divisors of $\rho_{r, s}: X_{r, s} \rightarrow Y_{r, s}$ intersect as in Figure 3. We denote the exceptional curves over the $D_{4}$-singular points $P(\alpha \beta)(\alpha \beta \in \mathscr{P})$ as in Figure 4 and denote the exceptional curves over the $A_{1}$-singular points $Q(\gamma)(\gamma \in 2)$ by $A(\gamma)$. The polarized $K 3$ surface $\left(X_{r, s}, H_{r, s}\right)$ has maximal rational double points. Consider the sublattice

$$
\Lambda_{r, s}:=\Lambda_{H} \oplus \bigoplus_{\alpha \beta \in \mathscr{P}} \Lambda_{P(\alpha \beta)} \oplus \bigoplus_{\gamma \in \mathcal{Q}} \Lambda_{Q(\gamma)}
$$

of $\mathrm{NS}\left(X_{r, s}\right)$ with finite index, as in Section 3C. The lattice $\Lambda_{H}$ is of rank 1 generated by $h:=\left[H_{r, s}\right]$, and $\Lambda_{H}^{\vee}$ is generated by $h^{\vee}:=h / 2$. The lattice $\Lambda_{P(\alpha \beta)}$ is of rank 4 with basis $d^{i}(\alpha \beta):=\left[D^{i}(\alpha \beta)\right](i=1, \ldots, 4)$. We denote the basis of $\Lambda_{P(\alpha \beta)}^{\vee}$ dual to $d^{1}(\alpha \beta), \ldots, d^{4}(\alpha \beta)$ by $d^{1}(\alpha \beta)^{\vee}, \ldots, d^{4}(\alpha \beta)^{\vee}$. The relations between $d^{1}(\alpha \beta), \ldots, d^{4}(\alpha \beta)$ and $d^{1}(\alpha \beta)^{\vee}, \ldots, d^{4}(\alpha \beta)^{\vee}$ are given by (2-4). The lattice $\Lambda_{Q(\gamma)}$ is of rank 1 generated by $a(\gamma):=[A(\gamma)]$, and $\Lambda_{Q(\gamma)}^{\vee}$ is generated

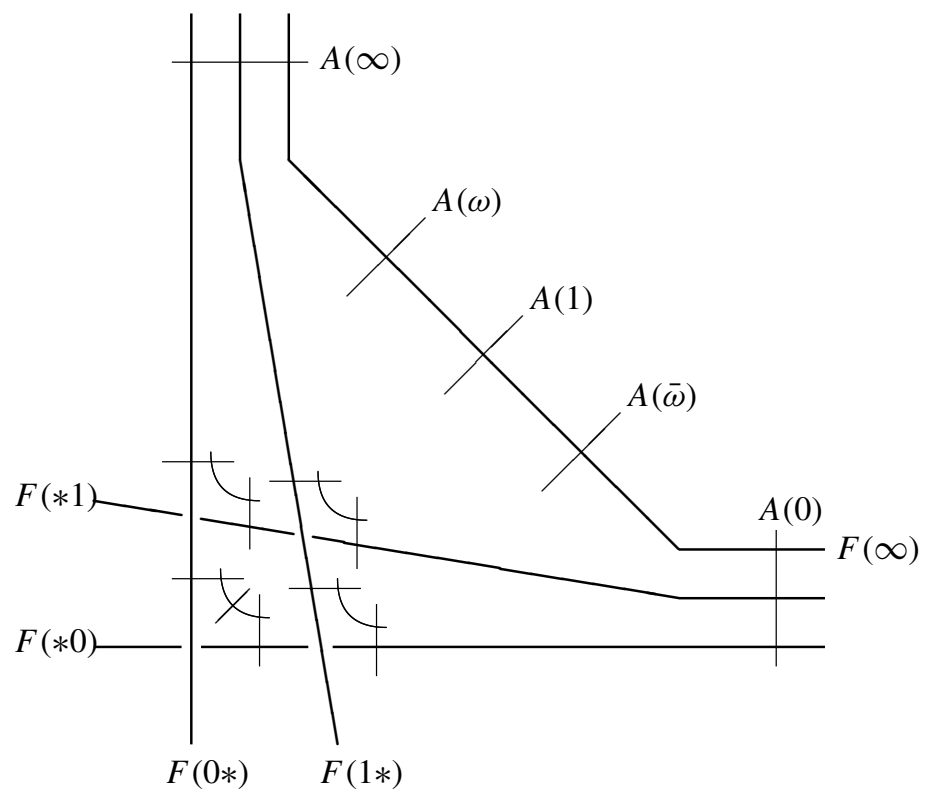

Figure 3. The configuration of half-lines and exceptional curves. 


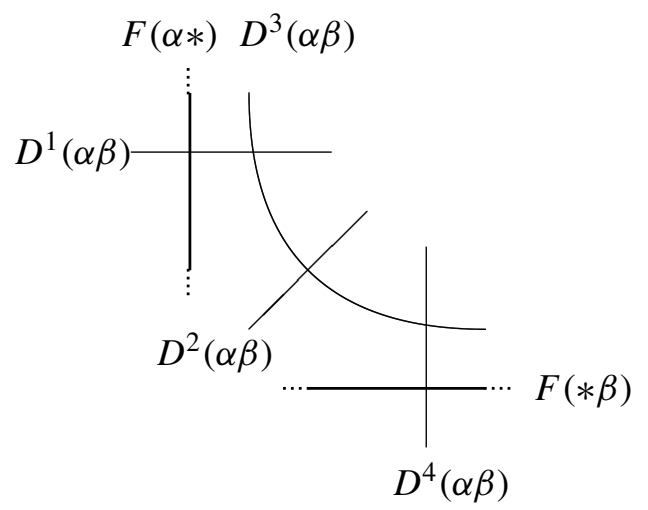

Figure 4. The exceptional curves over $P(\alpha \beta)$.

by $a(\gamma)^{\vee}:=-a(\gamma) / 2$. From Figures 3 and 4 , we see that the classes of halflines $F(\lambda)(\lambda \in \mathscr{L})$ are

$$
\begin{aligned}
& {[F(\infty)]=h^{\vee}+a(0)^{\vee}+a(\omega)^{\vee}+a(1)^{\vee}+a(\bar{\omega})^{\vee}+a(\infty)^{\vee},} \\
& {[F(0 *)]=h^{\vee}+d^{1}(00)^{\vee}+d^{1}(01)^{\vee}+a(\infty)^{\vee},} \\
& {[F(1 *)]=h^{\vee}+d^{1}(10)^{\vee}+d^{1}(11)^{\vee}+a(\infty)^{\vee},} \\
& {[F(* 0)]=h^{\vee}+d^{4}(00)^{\vee}+d^{4}(10)^{\vee}+a(0)^{\vee},} \\
& {[F(* 1)]=h^{\vee}+d^{4}(01)^{\vee}+d^{4}(11)^{\vee}+a(0)^{\vee} .}
\end{aligned}
$$

We then put

$$
\Delta_{r, s}:=\left(\Lambda_{r, s}\right)^{\vee} / \Lambda_{r, s}=\Delta_{H} \oplus \bigoplus_{\alpha \beta \in \mathscr{P}} \Delta_{P(\alpha \beta)} \oplus \bigoplus_{\gamma \in \mathcal{L}} \Delta_{Q(\gamma)},
$$

which is an $\mathbb{F}_{2}$-vector space of dimension 14. Since the discriminant of $\operatorname{NS}\left(X_{r, s}\right)$ is $-2^{2 \sigma\left(X_{r, s}\right)}=-2^{4}$, we see that $\operatorname{NS}\left(X_{r, s}\right) / \Lambda_{r, s} \subset \Delta_{r, s}$ is a subspace of dimension 5. It is easy to prove that the five elements

$$
\overline{[F(\lambda)]}:=[F(\lambda)] \bmod \Lambda_{r, s} \quad(\lambda \in \mathscr{L})
$$

of $\operatorname{NS}\left(X_{r, s}\right) / \Lambda_{r, s}$ are linearly independent. Therefore $\operatorname{NS}\left(X_{r, s}\right)$ is generated by the classes $h=\left[H_{r, s}\right], d^{i}(\alpha \beta)=\left[D^{i}(\alpha \beta)\right], a(\gamma)=[A(\gamma)]$, and $[F(\lambda)]$.

Remark 5.1. Suppose that $r^{3}=s^{3}$. Then there exists $c \in \mathbb{F}_{4}^{\times}=\{1, \omega, \bar{\omega}\}$ such that $s=c r$ holds. The three points $p(00), p(11)$ and $q(c)$ on $\mathbb{P}^{2}$ are collinear. Let $M$ be the line passing through these points. Then $M$ is a splitting line for $\left(X_{r, c r}, H_{r, c r}\right)$. Let $G$ be the half-line lying over $M$. By blowing up $Y_{r, c r}$ at the 
points $P(00), P(11)$ and $Q(c)$, we see that

$$
[G]=h^{\vee}+d^{2}(00)^{\vee}+d^{2}(11)^{\vee}+a(c)^{\vee} .
$$

Note that $d^{2}(\alpha \beta)^{\vee} \equiv d^{1}(\alpha \beta)^{\vee}+d^{4}(\alpha \beta)^{\vee} \bmod \Lambda_{P(\alpha \beta)}$ by (2-4). Hence $\overline{[G]}:=$ $[G] \bmod \Lambda_{r, c r}$ is linearly independent from the set of vectors $\overline{[F(\lambda)]}(\lambda \in \mathscr{L})$ in $\Delta_{r, c r}$. In particular, the linear subspace $\mathrm{NS}\left(X_{r, c r}\right) / \Lambda_{r, c r}$ of $\Delta_{r, c r}$ is of dimension 6 generated by $\overline{[F(\lambda)]}(\lambda \in \mathscr{L})$ and $\overline{[G]}$, and the Artin invariant of $X_{r, c r}$ is 1 .

\section{Proof of main theorem}

Note that supersingular $K 3$ surfaces with Artin invariant 1 are isomorphic to each other [Ogus 1983; Dolgachev and Kondō 2003]. Therefore it is enough to prove Theorem 1.2 under the additional assumption that the Artin invariant of $X^{\prime}$ is 2 .

We choose a Schröer's Kummer surface $X$ with $\sigma(X)=2$. To fix the ideas, we choose $s \in k \backslash \mathbb{F}_{4}$, put $X:=X_{1, s}$, and set

$$
H:=H_{1, s}, \quad Y:=Y_{1, s}, \quad \rho:=\rho_{1, s}, \quad \pi:=\pi_{1, s}, \quad \Delta:=\Delta_{1, s}, \quad \Lambda:=\Delta_{1, s} .
$$

Let $X^{\prime}$ be a supersingular $K 3$ surface with Artin invariant 2. Theorem 1.3 implies that $\mathrm{NS}(X)$ and $\mathrm{NS}\left(X^{\prime}\right)$ are isomorphic. By Proposition 3.1, there exists an isomorphism $\phi: \mathrm{NS}(X) \stackrel{\sim}{\rightarrow} \mathrm{NS}\left(X^{\prime}\right)$ such that $\phi \otimes \mathbb{R}$ maps $\operatorname{Nef}(X)$ to $\operatorname{Nef}\left(X^{\prime}\right)$. We fix such an isomorphism $\phi$ once and for all. By Corollary 3.4, we have a polarization $H^{\prime}$ of $X^{\prime}$ with degree 2 such that $\left[H^{\prime}\right]=\phi([H])$. As before, let

$$
X^{\prime} \stackrel{\rho^{\prime}}{\longrightarrow} Y^{\prime} \stackrel{\pi^{\prime}}{\longrightarrow} \mathbb{P}^{2}
$$

be the Stein factorization of $\Phi_{\left|H^{\prime}\right|}$. By Corollary 3.7, there exist bijections $\phi_{\mathscr{E}}$ : $\mathscr{E} \stackrel{\sim}{\rightarrow} \mathscr{E} \mathscr{E}^{\prime}$ and $\phi_{\text {Sing }}: \operatorname{Sing}(Y) \stackrel{\sim}{\rightarrow} \operatorname{Sing}\left(Y^{\prime}\right)$ such that the diagram (3-2) is commutative. For $P \in \operatorname{Sing}(Y)$, we write $P^{\prime} \in \operatorname{Sing}\left(Y^{\prime}\right)$ instead of $\phi_{\text {Sing }}(P)$, and for $E \in \mathscr{E}$, we write $E^{\prime} \in \mathscr{E}^{\prime}$ instead of $\phi_{\mathscr{E}}(E)$. Therefore $\operatorname{Sing}\left(Y^{\prime}\right)$ consists of four $D_{4}$-singular points $P(\alpha \beta)^{\prime}(\alpha \beta \in \mathscr{P})$ and five $A_{1}$-singular points $Q(\gamma)^{\prime}(\gamma \in 2)$. For example, the $(-2)$-curves contracted to $P(\alpha \beta)^{\prime}$ by $\rho^{\prime}$ are $D^{1}(\alpha \beta)^{\prime}, D^{2}(\alpha \beta)^{\prime}, D^{3}(\alpha \beta)^{\prime}$ and $D^{4}(\alpha \beta)^{\prime}$. We then put

$$
p(\alpha \beta)^{\prime}:=\pi^{\prime}\left(P(\alpha \beta)^{\prime}\right) \quad \text { and } \quad q(\gamma)^{\prime}:=\pi^{\prime}\left(Q(\gamma)^{\prime}\right) .
$$

We also set

$$
\Lambda^{\prime}:=\Lambda_{H^{\prime}} \oplus \bigoplus_{\alpha \beta \in \mathscr{P}} \Lambda_{P(\alpha \beta)^{\prime}} \oplus \bigoplus_{\gamma \in \mathscr{Q}} \Lambda_{Q(\gamma)^{\prime}}
$$

and

$$
\Delta^{\prime}:=\left(\Lambda^{\prime}\right)^{\vee} / \Lambda^{\prime}=\Delta_{H^{\prime}} \oplus \bigoplus_{\alpha \beta \in \mathscr{P}} \Delta_{P(\alpha \beta)^{\prime}} \oplus \bigoplus_{\gamma \in \mathscr{Q}} \Delta_{Q(\gamma)^{\prime}}
$$


as (5-1) and (5-3). Note that $\phi$ induces isomorphisms

$$
\phi_{\Lambda}: \Lambda \stackrel{\sim}{\rightarrow} \Lambda^{\prime} \text { and } \phi_{\Delta}: \Delta \stackrel{\sim}{\rightarrow} \Delta^{\prime}
$$

that are compatible with the direct-sum decompositions (5-1), (6-1), and (5-3), (6-2).

Let $L \subset \mathbb{P}^{2}$ be a line splitting in $(X, H)$, and let $F$ be the half-line of $(X, H)$ lying over $L$. We can define a line $L^{\prime} \subset \mathbb{P}^{2}$ splitting in $\left(X^{\prime}, H^{\prime}\right)$ and a half-line $F^{\prime}$ of $\left(X^{\prime}, H^{\prime}\right)$ lying over $L^{\prime}$ as follows.

Claim 6.1. There exists a unique effective divisor $D^{\prime}$ that represents $\phi([F])$.

Proof. Since $\phi([F])^{2}=-2$ and $\phi([F])\left[H^{\prime}\right]=1$, there exists an effective divisor $D^{\prime}$ that represents $\phi([F])$. Let $D^{\prime}=\Gamma^{\prime}+M^{\prime}$ be the decomposition of $D^{\prime}$ into the sum of the fixed part $\Gamma^{\prime}$ and the movable part $M^{\prime}$. Suppose that $M^{\prime} \neq 0$. If $M^{\prime} H^{\prime}=0$, then $M^{\prime 2}<0$ because $\left[H^{\prime}\right]^{\perp}$ is negative-definite. Therefore we have $M^{\prime} H^{\prime}>0$. Since $\Gamma^{\prime} H^{\prime} \geq 0$, we have $M^{\prime} H^{\prime}=1$, which implies that $\Phi_{\left|H^{\prime}\right|}$ induces an isomorphism from $M^{\prime}$ to a line on $\mathbb{P}^{2}$. Hence $M^{\prime}$ is a smooth rational curve, which is a contradiction.

Since $D^{\prime} H^{\prime}=1$, there exists a unique irreducible component $F^{\prime}$ of $D^{\prime}$ such that $F^{\prime} H^{\prime}=1$. Then $F^{\prime}$ is a half-line of $\left(X^{\prime}, H^{\prime}\right)$. We define $L^{\prime} \subset \mathbb{P}^{2}$ to be the image of $F^{\prime}$ by $\rho^{\prime} \circ \pi^{\prime}$.

Claim 6.2. Let $F^{\prime \prime}$ be a half-line for $\left(X^{\prime}, H^{\prime}\right)$ lying over $L^{\prime}$. Then $\overline{\left[F^{\prime \prime}\right]}=\overline{\left[F^{\prime}\right]}$ holds in $\Delta^{\prime}$, where $\overline{\left[F^{\prime \prime}\right]}=\left[F^{\prime \prime}\right] \bmod \Lambda^{\prime}$ and $\overline{\left[F^{\prime}\right]}=\left[F^{\prime}\right] \bmod \Lambda^{\prime}$.

Proof. The case where $F^{\prime}=F^{\prime \prime}$ is obvious. Suppose that $F^{\prime} \neq F^{\prime \prime}$. Then $F^{\prime}+F^{\prime \prime}$ is the total transform of $L^{\prime}$ in $X^{\prime}$ minus a linear combination of curves in $\mathscr{E}^{\prime}$, and hence $\left[F^{\prime}\right]+\left[F^{\prime \prime}\right] \in \Lambda^{\prime}$. Because $\Delta^{\prime}$ is a 2-elementary abelian group, we obtain $\overline{\left[F^{\prime \prime}\right]}=\overline{\left[F^{\prime}\right]}$.

Claim 6.3. We have $\phi_{\Delta}(\overline{[F]})=\overline{\left[F^{\prime}\right]}$.

Proof. Since $\phi([F])=\left[D^{\prime}\right]$, we have $\phi_{\Delta}(\overline{[F]})=\overline{\left[D^{\prime}\right]}$. Since $D^{\prime}-F^{\prime}$ is effective or zero and $\left(D^{\prime}-F^{\prime}\right) H^{\prime}=0$, each irreducible component of $D^{\prime}-F^{\prime}$ is contracted to a point by $\rho^{\prime}$. Therefore we have $\left[D^{\prime}\right]-\left[F^{\prime}\right] \in \Lambda^{\prime}$, and hence $\overline{\left[D^{\prime}\right]}=\overline{\left[F^{\prime}\right]}$.

Now we have half-lines $F(\lambda)^{\prime}$ and splitting lines $L(\lambda)^{\prime}$ of $\left(X^{\prime}, H^{\prime}\right)$ for each $\lambda \in \mathscr{L}$. By Claim 6.3, the elements $\overline{\left[F(\lambda)^{\prime}\right]}$ of $\Delta^{\prime}$ are distinct from each other. Hence, by Claim 6.2, the lines $L(\lambda)^{\prime}$ are distinct from each other.

Claim 6.4. Let $P$ be a point of $\operatorname{Sing}(Y)$. If $\pi(P) \in L(\lambda)$, then $\pi^{\prime}\left(P^{\prime}\right) \in L(\lambda)^{\prime}$.

Proof. If $\pi(P) \in L(\lambda)$, then the $P$-component of $\overline{[F(\lambda)]} \in \Delta$ is not zero by (5-2). Hence the $P^{\prime}$-component of $\overline{\left[F(\lambda)^{\prime}\right]} \in \Delta^{\prime}$ is not zero by Claim 6.3. Consequently, there exists $E^{\prime} \in \mathscr{E}_{P^{\prime}}^{\prime}$, such that $F(\lambda)^{\prime} E^{\prime} \neq 0$. Therefore the image $L(\lambda)^{\prime}$ of $F(\lambda)^{\prime}$ passes through $\pi^{\prime}\left(P^{\prime}\right) \in \mathbb{P}^{2}$. 
Claim 6.5. The splitting line $L(\lambda)^{\prime}$ is of nonreduced type for any $\lambda \in \mathscr{L}$.

Proof. Let $G^{\prime}$ be an arbitrary half-line of $\left(X^{\prime}, H^{\prime}\right)$ lying over $L(\lambda)^{\prime}$. Then the class $g^{\prime}:=\left[G^{\prime}\right] \in \mathrm{NS}\left(X^{\prime}\right)$ satisfies:
(i) $\left(g^{\prime}\right)^{2}=-2$;
(ii) $g^{\prime}\left[H^{\prime}\right]=1$;
(iii) $g^{\prime}\left[E^{\prime}\right] \geq 0$ for every $E^{\prime} \in \mathscr{E}^{\prime}$.

Suppose that $L(\lambda)^{\prime}$ is of reduced type. Then there exists a half-line $F^{\prime \prime}$ lying over $L(\lambda)^{\prime}$ that is distinct from $F(\lambda)^{\prime}$. Since $\left[F^{\prime \prime}\right]\left[F(\lambda)^{\prime}\right] \geq 0$, we have $\left[F^{\prime \prime}\right] \neq\left[F(\lambda)^{\prime}\right]$. By Claim 6.2, we have $\overline{\left[F(\lambda)^{\prime}\right]}=\overline{\left[F^{\prime \prime}\right]}$ in $\Delta^{\prime}$. Consequently, it is enough to show that there exists only one class $g^{\prime}$ in $\mathrm{NS}\left(X^{\prime}\right)$ satisfying (i), (ii), (iii) above and (iv) $\overline{\left(g^{\prime}\right)}=\overline{\left[F(\lambda)^{\prime}\right]}=\phi_{\Delta}(\overline{[F(\lambda)]})$,

where the second equality follows from Claim 6.3. We denote by $g_{H^{\prime}}^{\prime}$ and $g_{P^{\prime}}^{\prime}$ the $H^{\prime}$ - and $P^{\prime}$-components of $g^{\prime}$, respectively, where $P^{\prime} \in \operatorname{Sing}\left(Y^{\prime}\right)$. By (ii), we have $g_{H^{\prime}}^{\prime}=\left[H^{\prime}\right] / 2$. Combining this with (i), we have

$$
\sum_{\alpha \beta \in \mathscr{P}}\left(g_{P(\alpha \beta)^{\prime}}^{\prime}\right)^{2}+\sum_{\gamma \in \mathcal{L}}\left(g_{Q(\gamma)^{\prime}}^{\prime}\right)^{2}=-5 / 2 .
$$

The case where $\lambda=\infty$. By (iii), (iv), (5-2) and Lemmas 2.4, 2.5, we have

$$
\left(g_{P(\alpha \beta)^{\prime}}^{\prime}\right)^{2}=0 \text { or } \leq-2 \text { and }\left(g_{Q(\gamma)^{\prime}}^{\prime}\right)^{2}=-1 / 2 \text { or } \leq-9 / 2 .
$$

Combining this with (6-3), we have

$$
\left(g_{P(\alpha \beta)^{\prime}}^{\prime}\right)^{2}=0 \quad \text { and } \quad\left(g_{Q(\gamma)^{\prime}}^{\prime}\right)^{2}=-1 / 2 .
$$

By (iii) and Lemmas 2.4, 2.5 again, we have

$$
g_{P(\alpha \beta)^{\prime}}^{\prime}=0 \quad \text { and } \quad g_{Q(\gamma)^{\prime}}^{\prime}=-\left[A(\gamma)^{\prime}\right] / 2 .
$$

Thus the uniqueness of $g^{\prime}$ is proved.

The case where $\lambda=0 *$. By (iii), (iv), (5-2) and Lemmas 2.4, 2.5, we have

$$
\begin{array}{ll}
\left(g_{P(\alpha \beta)^{\prime}}^{\prime}\right)^{2}=-1 \text { or } \leq-3, & \text { if } \alpha \beta=00 \text { or } 01, \\
\left(g_{P(\alpha \beta)^{\prime}}^{\prime}\right)^{2}=0 \text { or } \leq-2, & \text { if } \alpha \beta=10 \text { or } 11, \\
\left(g_{Q(\gamma)^{\prime}}^{\prime}\right)^{2}=-1 / 2 \text { or } \leq-9 / 2, & \text { if } \gamma=\infty, \\
\left(g_{Q(\gamma)^{\prime}}^{\prime}\right)^{2}=0 \text { or } \leq-2, & \text { if } \gamma \neq \infty .
\end{array}
$$

Combining this with (6-3), we have

$$
\begin{array}{ll}
\left(g_{P(00)^{\prime}}^{\prime}\right)^{2}=\left(g_{P(01)^{\prime}}^{\prime}\right)^{2}=-1, & \left(g_{Q(\infty)^{\prime}}^{\prime}\right)^{2}=-1 / 2, \\
\left(g_{P(10)^{\prime}}^{\prime}\right)^{2}=\left(g_{P(11)^{\prime}}^{\prime}\right)^{2}=0, & \left(g_{Q(\gamma)^{\prime}}^{\prime}\right)^{2}=0, \quad \text { for } \gamma \neq \infty .
\end{array}
$$


By (iii) and Lemmas 2.4, 2.5 again, we have

$$
\begin{aligned}
& g_{P(00)^{\prime}}^{\prime}=\delta^{1}(00), \\
& g_{P(01)^{\prime}}^{\prime}=\delta^{1}(01), \\
& g_{P(10)^{\prime}}^{\prime}=g_{P(11)^{\prime}}^{\prime}=0 \text {, } \\
& \begin{aligned}
g_{Q(\infty)^{\prime}}^{\prime} & =-\left[A(\infty)^{\prime}\right] / 2, \\
g_{Q(\gamma)^{\prime}}^{\prime} & =0, \quad \text { for } \gamma \neq \infty,
\end{aligned}
\end{aligned}
$$

where

$$
\delta^{1}(\alpha \beta)=-\left[D^{1}(\alpha \beta)^{\prime}\right]-\left[D^{2}(\alpha \beta)^{\prime}\right] / 2-\left[D^{3}(\alpha \beta)^{\prime}\right]-\left[D^{4}(\alpha \beta)^{\prime}\right] / 2 .
$$

See (2-4). Thus the uniqueness of $g^{\prime}$ is proved.

The other cases $\lambda=1 *, * 0, * 1$ can be treated in the same way.

We have five distinct splitting lines $L(\lambda)^{\prime}(\lambda \in \mathscr{L})$ for $\left(X^{\prime}, H^{\prime}\right)$, which are of nonreduced type by Claim 6.5. By Lemma 4.3, we see that $\pi^{\prime}: Y^{\prime} \rightarrow \mathbb{P}^{2}$ is purely inseparable. By Claim 6.4, the configuration of the lines $L(\lambda)^{\prime}$ and the points $p(\alpha \beta)^{\prime}, q(\gamma)^{\prime}$ are exactly the same as the configuration depicted in Figure 2 with superscript prime $\left(^{\prime}\right)$ being put to everything.

There exists a homogeneous coordinate system $[x: y: z]$ of $\mathbb{P}^{2}$ such that

$$
\begin{array}{rlrl}
q(\infty)^{\prime} & =[0: 1: 0], & & p(00)^{\prime}=[0: 0: 1], \\
q(1)^{\prime}=[1: 1: 0], & & p(10)^{\prime}=[1: 0: 1] . \\
q(0)^{\prime}=[1: 0: 0], & &
\end{array}
$$

We put

$$
p(01)^{\prime}=[0: t: 1],
$$

where $t$ is a nonzero constant. Then we have $p(11)^{\prime}=[1: t: 1]$ by Figure' 2 . Let

$$
w^{2}=G(x, y, z)
$$

be the defining equation of $Y^{\prime}$, where $G$ is a homogeneous polynomial of degree 6, and let $G_{l m n}(l+m+n=6)$ be the coefficient of $x^{l} y^{m} z^{n}$ in $G$. By Remark 4.4, we can assume

$$
G_{l m n}=0 \quad \text { if } \quad l \equiv m \equiv n \equiv 0 \bmod 2 .
$$

Using Lemma 4.5(1), we obtain:

$$
\begin{array}{ll}
G_{015}=G_{033}=G_{051}=0, & \text { because } L(0 *)^{\prime}=\{x=0\} \text { is splitting; } \\
G_{105}=G_{303}=G_{501}=0, & \text { because } L(* 0)^{\prime}=\{y=0\} \text { is splitting; } \\
G_{150}=G_{330}=G_{510}=0, & \text { because } L(\infty)^{\prime}=\{z=0\} \text { is splitting. }
\end{array}
$$

Therefore

$$
G(x, y, z)=x y z C(x, y, z)
$$


where $C$ is a homogeneous polynomial of degree 3. By Lemma 4.5(2), the line $L(0 *)^{\prime}=\{x=0\}$ and the quintic curve defined by $y z C(x, y, z)=0$ intersect transversely at $q(\infty)^{\prime}$ and with multiplicity $\geq 2$ at $p(00)^{\prime}$ and $p(01)^{\prime}$. Therefore there exists a constant $A$ such that $y z C(0, y, z)=A y^{2} z(y+t z)^{2}$. In particular, we obtain

$$
G_{132}=G_{114}=0 \text { and } G_{123}=t^{2} G_{141} .
$$

By Lemma 4.5(2), the line $L(* 0)^{\prime}=\{y=0\}$ and the curve $x z C(x, y, z)=0$ intersect transversely at $q(0)^{\prime}$ and with multiplicity $\geq 2$ at $p(00)^{\prime}$ and $p(10)^{\prime}$. Therefore there exists a constant $B$ such that $x z C(x, 0, z)=B x^{2} z(x+z)^{2}$. In particular, we obtain

$$
G_{312}=G_{114}=0 \quad \text { and } \quad G_{213}=G_{411} .
$$

By Lemma 4.5(2), the line $L(\infty)^{\prime}=\{z=0\}$ and the curve $x y C(x, y, z)=0$ intersect transversely at the five points $q(\gamma)^{\prime}(\gamma \in 2)$. In particular, the curve $x y C(x, y, z)=0$ passes through $q(1)^{\prime}$, and hence we obtain

$$
G_{141}+G_{231}+G_{321}+G_{411}=0 .
$$

Combining these, we see that $Y^{\prime}$ is defined by

$$
w^{2}=x y z\left(t^{2} a y z^{2}+d x z^{2}+a y^{3}+b x y^{2}+c x^{2} y+d x^{3}\right),
$$

where $a, b, c, d$ are constants such that $a+b+c+d=0$. Because $L(1 *)^{\prime}=\{x=z\}$ is splitting, the polynomial $y z^{2}\left(t^{2} y z^{2}+a y^{3}+b z y^{2}+c z^{2} y\right)$ of $y$ and $z$ is the square of a cubic polynomial. Therefore $b=0$. Because $L(* 1)^{\prime}=\{y=t z\}$ is splitting, the polynomial $t x z^{2}\left(d x z^{2}+b t^{2} x z^{2}+c t x^{2} z+d x^{3}\right)$ of $x$ and $z$ is the square of a cubic polynomial. Therefore $c=0$. Because $a+b+c+d=0$, we have $a=d$. Therefore $Y^{\prime}$ is defined by

$$
w^{2}=x y z\left(t^{2} y z^{2}+x z^{2}+y^{3}+x^{3}\right) .
$$

Hence $Y^{\prime}$ is isomorphic to Schröer's normal $K 3$ surface $Y_{t, 1}$, and hence $X^{\prime}$ is isomorphic to Schröer's Kummer surface $X_{t, 1}$.

Remark 6.6. In [Pho and Shimada 2006], it is shown that every supersingular $K 3$ surface in characteristic 5 with Artin invariant $\leq 3$ is obtained as a double cover of the projective plane with the branch curve defined by $y^{5}-f(x)=0$, where $f(x)$ is a polynomial of degree 6 , and hence it is unirational.

\section{References}

[Artin 1962] M. Artin, "Some numerical criteria for contractability of curves on algebraic surfaces", Amer. J. Math. 84 (1962), 485-496. MR 26 \#3704 Zbl 0105.14404

[Artin 1966] M. Artin, "On isolated rational singularities of surfaces", Amer. J. Math. 88 (1966), 129-136. MR 33 \#7340 Zbl 0142.18602 
[Artin 1974] M. Artin, "Supersingular K3 surfaces", Ann. Sci. École Norm. Sup. (4) 7 (1974), 543567. MR 51 \#8116 Zbl 0322.14014

[Artin 1977] M. Artin, "Coverings of the rational double points in characteristic p", pp. 11-22 in Complex analysis and algebraic geometry, Iwanami Shoten, Tokyo, 1977. MR 56 \#8559 Zbl 0358. 14008

[Blass and Lang 1987] P. Blass and J. Lang, Zariski surfaces and differential equations in characteristic $p>0$, Pure and Applied Mathematics 106, Marcel Dekker, New York, 1987. MR 88g:14032 Zbl 0614.14011

[Bourbaki 1981] N. Bourbaki, Éléments de mathématique; Groupes et algèbres de Lie. Chapitres 4, 5 et 6, Masson, Paris, 1981. MR 83g:17001 Zbl 0498.12001

[Dolgachev and Kondō 2003] I. Dolgachev and S. Kondō, "A supersingular K3 surface in characteristic 2 and the Leech lattice”, Int. Math. Res. Not. 1 (2003), 1-23. MR 2003i:14051 Zbl 1061.14031

[Ebeling 2002] W. Ebeling, Lattices and codes, revised ed., Advanced Lectures in Mathematics, Friedr. Vieweg \& Sohn, Braunschweig, 2002. MR 2003i:11093 Zbl 1030.11030

[Greuel and Kröning 1990] G.-M. Greuel and H. Kröning, "Simple singularities in positive characteristic”, Math. Z. 203:2 (1990), 339-354. MR 90k:14001 Zbl 0715.14001

[Katsura 1978] T. Katsura, "On Kummer surfaces in characteristic 2”, pp. 525-542 in Proceedings of the International Symposium on Algebraic Geometry (Kyoto, 1977), edited by M. Nagata, Kinokuniya Book Store, Tokyo, 1978. MR 82d:14022 Zbl 0411.14010

[Nikulin 1991] V. V. Nikulin, "Weil linear systems on singular K3 surfaces", pp. 138-164 in Algebraic geometry and analytic geometry (Tokyo, 1990), edited by A. Fujiki et al., ICM-90 Satell. Conf. Proc., Springer, Tokyo, 1991. MR 94k:14029 Zbl 0785.14021

[Ogus 1979] A. Ogus, "Supersingular K3 crystals", pp. 3-86 in Journées de Géométrie Algébrique de Rennes II (Rennes, 1978), Astérisque 64, Soc. Math. France, Paris, 1979. MR 81e:14024 Zbl 0435.14003

[Ogus 1983] A. Ogus, "A crystalline Torelli theorem for supersingular K3 surfaces”, pp. 361-394 in Arithmetic and geometry, Vol. II, edited by M. Artin and J. Tate, Progr. Math. 36, Birkhäuser, Boston, 1983. MR 85d:14055 Zbl 0596.14013

[Pho and Shimada 2006] D. T. Pho and I. Shimada, "Unirationality of certain supersingular K3 surfaces in characteristic 5", Manuscripta Math. 121:4 (2006), 425-435. MR 2007i:14036 Zbl 1108.14029

[Rudakov and Shafarevich 1981] A. N. Rudakov and I. R. Shafarevich, "Surfaces of type K3 over fields of finite characteristic", pp. 115-207 in Current problems in mathematics, vol. 18, Akad. Nauk SSSR, Vsesoyuz. Inst. Nauchn. i Tekhn. Informatsii, Moscow, 1981. In Russian; translated in Collected mathematical papers, Springer, Berlin, 1989. MR 83c:14027 Zbl 0669.12001

[Saint-Donat 1974] B. Saint-Donat, "Projective models of K-3 surfaces", Amer. J. Math. 96 (1974), 602-639. MR 51 \#518 Zbl 0301.14011

[Schröer 2007] S. Schröer, "Kummer surfaces for the self-product of the cuspidal rational curve", J. Algebraic Geom. 16:2 (2007), 305-346. MR 2007i:14038 Zbl 05161022

[Shimada 2004a] I. Shimada, "Rational double points on supersingular K3 surfaces", Math. Comp. 73:248 (2004), 1989-2017. MR 2005g:14076 Zbl 1052.14041

[Shimada 2004b] I. Shimada, "Supersingular K3 surfaces in characteristic 2 as double covers of a projective plane", Asian J. Math. 8:3 (2004), 531-586. MR 2006b:14064 Zbl 1080.14047

[Shimada 2006] I. Shimada, "Moduli curves of supersingular K3 surfaces in characteristic 2 with Artin invariant 2", Proc. Edinb. Math. Soc. (2) 49:2 (2006), 435-503. MR 2007h:14051 Zbl 1101. 14053 
[Shimada and Zhang 2007] I. Shimada and D.-Q. Zhang, "K3 surfaces with ten cusps", pp. 187-211 in Algebraic geometry (Seoul, 2004), edited by J. Keum and S. Kondō, Contemp. Math. 422, Amer. Math. Soc., Providence, RI, 2007. MR MR2296438 Zbl 05155374

[Shioda 1974] T. Shioda, "Kummer surfaces in characteristic 2", Proc. Japan Acad. 50 (1974), 718722. MR 58 \#10929 Zbl 0332.14015

[Shioda 1979] T. Shioda, "Supersingular K3 surfaces", pp. 564-591 in Algebraic geometry (Copenhagen, 1978), edited by K. Lønsted, Lecture Notes in Mathematics 732, Springer, Berlin, 1979. MR 82c:14030 Zbl 0414.14019

[Urabe 1988] T. Urabe, "Combinations of rational singularities on plane sextic curves with the sum of Milnor numbers less than sixteen”, pp. 429-456 in Singularities (Warsaw, 1985), edited by S. Łojasiewicz, Banach Center Publ. 20, PWN, Warsaw, 1988. MR 92g:14025 Zbl 0674.14001

Received June 22, 2006.

\author{
ICHIRO SHIMADA \\ DiVISION OF MATHEMATICS \\ GraduATE SCHOOL OF SCIENCE \\ HOKKAIDO UNIVERSITY \\ SAPPORO 060-0810 \\ JAPAN \\ shimada@math.sci.hokudai.ac.jp \\ http://www.math.sci.hokudai.ac.jp/ shimada/ \\ DE-QI ZHANG \\ DEPARTMENT OF MATHEMATICS \\ NATIONAL UNIVERSITY OF SINGAPORE \\ LOWER KENT RIDGE ROAD \\ SINGAPORE 119260 \\ matzdq@math.nus.edu.sg
}

